\title{
ON COVERINGS OF SIMPLE ABELIAN VARIETIES
}

\author{
BY OLIVIER DEBARRE
}

\begin{abstract}
To any finite covering $f: Y \rightarrow X$ of degree $d$ between smooth complex projective manifolds, one associates a vector bundle $E_{f}$ of rank $d-1$ on $X$ whose total space contains $Y$. It is known that $E_{f}$ is ample when $X$ is a projective space ([9]), a Grassmannian ([11]), or a Lagrangian Grassmannian ([7]). We show an analogous result when $X$ is a simple abelian variety and $f$ does not factor through any nontrivial isogeny $X^{\prime} \rightarrow X$. This result is obtained by showing that $E_{f}$ is $M$-regular in the sense of Pareschi-Popa, and that any $M$-regular sheaf is ample.

RÉSumÉ (Sur les revêtements des variétés abéliennes simples). - On associe à tout revêtement fini $f: Y \rightarrow X$ de degré $d$ entre variétés projectives lisses complexes un fibré vectoriel $E_{f}$ de rang $d-1$ sur $X$ dont l'espace total contient $Y$. On sait que $E_{f}$ est ample lorsque $X$ est un espace projectif ([9]), une grassmannienne ([11]) ou une grassmannienne lagrangienne ([7]). Nous montrons un résultat analogue lorsque $X$ est une variété abélienne simple et que $f$ ne se factorise par aucune isogénie non triviale $X^{\prime} \rightarrow X$. Ce résultat est obtenu en montrant que $E_{f}$ est $M$-régulier au sens de Pareschi-Popa, puis que tout faisceau $M$-régulier est ample.
\end{abstract}

Texte reçu le 1er octobre 2004, accepté le 2 février 2005.

Olivier Debarre, UFR de Mathématique et d'Informatique, 7 rue René Descartes, 67084 Strasbourg Cedex (France). - E-mail : debarre@math.u-strasbg.fr 2000 Mathematics Subject Classification. — 14E20, 14J60, 14K02, 14K05, 14 K12.

Key words and phrases. - Abelian variety, vector bundle, ample sheaf, $M$-regular sheaf, continuously generated sheaf, Barth-Lefschetz Theorem, Mukai transform. 


\section{Introduction}

We work over the complex numbers. Let $f: Y \rightarrow X$ be a finite surjective morphism of degree $d$ between smooth projective varieties of the same dimension $n$. The morphism $f$ is flat, hence the sheaf $f_{*} \mathcal{O}_{Y}$ is locally free. We may define a locally free sheaf $E_{f}$ of rank $d-1$ on $X$ as the dual of the kernel of the trace map $\operatorname{Tr}_{Y / X}: f_{*} \mathcal{O}_{Y} \rightarrow \mathcal{O}_{X}$, so that

$$
f_{*} \mathcal{O}_{Y}=\mathcal{O}_{X} \oplus E_{f}^{*}
$$

By duality for a finite flat morphism, we have

$$
f_{*} \omega_{Y / X}=\mathcal{O}_{X} \oplus E_{f}
$$

Our aim is to prove the following statement conjectured in [1].

THEOREM 1.1. - Let $X$ be a simple abelian variety, let $Y$ be a smooth connected projective variety, and let $f: Y \rightarrow X$ be a finite cover. If $f$ does not factor through any nontrivial isogeny $X^{\prime} \rightarrow X$, the vector bundle $E_{f}$ is ample.

For a more general statement, see Theorem 4.1. See also the remarks at the end of this article for more comments. Even if $X$ is not simple, the vector bundle $E_{f}$ is known to be nef (see [14, Theorem 1.17], [10, Example 6.3.59]) and its restriction to a general complete intersection curve in $X$ to be ample (see [6, Lemma 2.7]).

The ampleness of $E_{f}$ has a number of consequences, as explained in [10, Example 6.3.56]. In our case, one new statement beyond the Fulton-Hansentype results already obtained in [1] is the following: under the hypotheses of the theorem, the induced morphism

$$
H^{i}(f, \mathbb{C}): H^{i}(X, \mathbb{C}) \longrightarrow H^{i}(Y, \mathbb{C})
$$

is bijective for $i \leq n-d+1$ (see [10, Theorem 7.1.16]).

When moreover $d \leq n$, the morphism $\pi_{1}(f): \pi_{1}(Y) \rightarrow \pi_{1}(X)$ is bijective. ${ }^{(1)}$ In particular, the group $H_{1}(Y, \mathbb{Z})$ is isomorphic to $H_{1}(X, \mathbb{Z})$, hence is torsionfree, and so is $H^{2}(Y, \mathbb{Z})$ by the universal coefficient theorem.

When $d \leq n-1$, the morphism $H^{2}(f, \mathbb{Z}): H^{2}(X, \mathbb{Z}) \rightarrow H^{2}(Y, \mathbb{Z})$ is injective with finite cokernel, hence so is $\operatorname{Pic}(f): \operatorname{Pic}(X) \rightarrow \operatorname{Pic}(Y)$. It seems likely that those two maps are bijective.

The proof is a simple application of the results of [13] about global generation of sheaves on an abelian variety. More precisely, it is based on the remark that any $M$-regular sheaf ( $(3)$ on an abelian variety is ample (Corollary 3.2 ).

(1) For algebraic fundamental groups, this is [1, Corollaire 6.2]; for topological fundamental groups, this is [2, Exercice VIII.5], where the hypothesis $d \leq n$ is unfortunately missing.

TOME $134-2006-\mathrm{N}^{\mathrm{O}} 2$ 
This work was done while the author was visiting the University of Michigan at Ann Arbor. Many thanks to Bill Fulton and Rob Lazarsfeld for support and many stimulating conversations.

\section{Ample sheaves}

To any coherent sheaf $\mathcal{F}$ on a scheme $X$ of finite type over $\mathbb{C}$, one associates the $X$-scheme

$$
\mathbf{P}(\mathcal{F})=\operatorname{Proj}\left(\bigoplus_{m \geq 0} \operatorname{Sym}^{m} \mathcal{F}\right)
$$

and an invertible sheaf $\mathcal{O}_{\mathbf{P}(\mathcal{F})}(1)$ on $\mathbf{P}(\mathcal{F})$. The sheaf $\mathcal{F}$ is said to be ample if $\mathcal{O}_{\mathbf{P}(\mathcal{F})}(1)$ is.

Well-known properties of ampleness for locally free sheaves (see for example [10, Chapter 6]) still hold in this general setting:

a) the sheaf $\mathcal{F}$ is ample if and only if, for any coherent sheaf $\mathcal{G}$ on $X$, the sheaf $\mathcal{G} \otimes \mathbf{S y m}^{m} \mathcal{F}$ is globally generated for all $m \gg 0$ (see [8, Theorem 1]);

b) any quotient of an ample sheaf is ample (see [8, Proposition 1]);

c) if $\pi: Y \rightarrow X$ is a finite morphism, $\mathcal{F}$ is ample if and only if $\pi^{*} \mathcal{F}$ is (this is because $\mathbf{P}\left(\pi^{*} \mathcal{F}\right)=\mathbf{P}(\mathcal{F}) \times_{X} Y$ and $\mathcal{O}_{\mathbf{P}(\mathcal{F})}(1)$ pulls back, by a finite morphism, to $\left.\mathcal{O}_{\mathbf{P}\left(\pi^{*} \mathcal{F}\right)}(1)\right)$

d) if $X$ is proper and $\mathcal{F}$ is globally generated, $\mathcal{F}$ is ample if and only if, for any curve $C$ in $X$, the restriction $\mathcal{F} \otimes \mathcal{O}_{C}$ has no trivial quotient (Gieseker's Lemma).

\section{Continuously generated sheaves}

Following [13, Definition 2.10], we say that a coherent sheaf $\mathcal{F}$ on an irreducible projective variety $X$ is continuously globally generated if, for any nonempty subset $U$ of $\operatorname{Pic}^{0}(X)$, the sum of the twisted evaluation maps

$$
\bigoplus_{\xi \in U} H^{0}\left(X, \mathcal{F} \otimes P_{\xi}\right) \otimes P_{\xi}^{\vee} \longrightarrow \mathcal{F}
$$

is surjective, where, for any element $\xi$ of $\operatorname{Pic}^{0}(X)$, we denote by $P_{\xi}$ the corresponding numerically trivial line bundle on $X$. This property is equivalent to the existence of a positive integer $N$ such that for $\left(\xi_{1}, \ldots, \xi_{N}\right)$ general in $\operatorname{Pic}^{0}(X)^{N}$, the analogous map

$$
\bigoplus_{i=1}^{N} H^{0}\left(X, \mathcal{F} \otimes P_{\xi_{i}}\right) \otimes P_{\xi_{i}}^{\vee} \longrightarrow \mathcal{F}
$$

BULletin DE LA SOCiÉtÉ MATHÉmATiQUe DE FRANCE 
is surjective. Being a quotient of a direct sum of numerically trivial line bundles, a continuously globally generated sheaf is nef. Our aim is to show that under certain circumstances, it is ample.

Proposition 3.1. - A coherent sheaf $\mathcal{F}$ on an irreducible projective variety $X$ is continuously globally generated if and only if there exists a connected abelian Galois étale cover $\pi: Y \rightarrow X$ such that $\pi^{*}\left(\mathcal{F} \otimes P_{\xi}\right)$ is globally generated for all $\xi \in \operatorname{Pic}^{0}(X)$.

Proof. - Assume $\mathcal{F}$ is continuously globally generated and let $\xi \in \operatorname{Pic}^{0}(X)$. Since torsion points are dense in $\operatorname{Pic}^{0}(X)^{N}$, the open subset of $\operatorname{Pic}^{0}(X)^{N}$ of points for which the map (1) is surjective and all $h^{0}\left(X, \mathcal{F} \otimes P_{\xi_{i}}\right)$ are minimal contains a point of the type

$$
\left(\xi+\eta_{1}(\xi), \ldots, \xi+\eta_{N}(\xi)\right)
$$

where $\left(\eta_{1}(\xi), \ldots, \eta_{N}(\xi)\right)$ is torsion, hence contains also $U_{\xi}+\left(\eta_{1}(\xi), \ldots, \eta_{N}(\xi)\right)$, where $U_{\xi}$ is a neighborhood of $\xi$ in $\operatorname{Pic}^{0}(X)$. Since $\operatorname{Pic}^{0}(X)$ is quasi-compact, it is covered by finitely many such neighborhoods, say $U_{\xi_{1}}, \ldots, U_{\xi_{M}}$.

Let $\pi: Y \rightarrow X$ be a connected abelian Galois étale cover such that the kernel of $\operatorname{Pic}^{0}(\pi): \operatorname{Pic}^{0}(X) \rightarrow \operatorname{Pic}^{0}(Y)$ contains all $\eta_{i}\left(\xi_{j}\right)$, for $i \in\{1, \ldots, N\}$ and $j \in\{1, \ldots, M\}$. Fix $j \in\{1, \ldots, M\}$; the map

$$
\bigoplus_{i=1}^{N} H^{0}\left(X, \mathcal{F} \otimes P_{\xi} \otimes P_{\eta_{i}\left(\xi_{j}\right)}\right) \otimes \pi^{*} P_{\xi}^{\vee} \otimes \pi^{*} P_{\eta_{i}\left(\xi_{j}\right)}^{\vee} \longrightarrow \pi^{*} \mathcal{F}
$$

is surjective for all $\xi \in U_{\xi_{j}}$. But this map is

$$
\bigoplus_{i=1}^{N} H^{0}\left(X, \mathcal{F} \otimes P_{\xi} \otimes P_{\eta_{i}\left(\xi_{j}\right)}\right) \otimes \pi^{*} P_{\xi}^{\vee} \longrightarrow \pi^{*} \mathcal{F}
$$

and since each $H^{0}\left(X, \mathcal{F} \otimes P_{\xi} \otimes P_{\eta_{i}\left(\xi_{j}\right)}\right)$ is a vector subspace of $H^{0}\left(Y, \pi^{*}\left(\mathcal{F} \otimes P_{\xi}\right)\right)$, the sheaf $\pi^{*}\left(\mathcal{F} \otimes P_{\xi}\right)$ is globally generated for all $\xi \in U_{\xi_{j}}$, hence for all $\xi$ in $\operatorname{Pic}^{0}(X)$.

For the converse, assume that there exists a connected abelian Galois étale cover $\pi: Y \rightarrow X$ such that the evaluation map

$$
H^{0}\left(Y, \pi^{*}\left(\mathcal{F} \otimes P_{\xi}\right)\right) \otimes \mathcal{O}_{Y} \longrightarrow \pi^{*}\left(\mathcal{F} \otimes P_{\xi}\right)
$$

is surjective for all $\xi \in \operatorname{Pic}^{0}(X)$. Since $\pi$ is finite, the map

$$
H^{0}\left(X, \mathcal{F} \otimes P_{\xi} \otimes \pi_{*} \mathcal{O}_{Y}\right) \otimes \pi_{*} \mathcal{O}_{Y} \longrightarrow \mathcal{F} \otimes P_{\xi} \otimes \pi_{*} \mathcal{O}_{Y}
$$

is also surjective. If we let $\operatorname{Ker}\left(\operatorname{Pic}^{0}(\pi)\right)=\left\{\eta_{1}, \ldots, \eta_{N}\right\}$, we have $\pi_{*} \mathcal{O}_{Y}=$ $\bigoplus_{i=1}^{N} P_{\eta_{i}}$, the map

$$
\left(\bigoplus_{i=1}^{N} H^{0}\left(X, \mathcal{F} \otimes P_{\xi} \otimes P_{\eta_{i}}\right)\right) \otimes\left(\bigoplus_{i=1}^{N} P_{\eta_{i}}\right) \longrightarrow \mathcal{F} \otimes P_{\xi} \otimes\left(\bigoplus_{i=1}^{N} P_{\eta_{i}}\right)
$$

TOME $134-2006-\mathrm{N}^{\mathrm{O}} 2$ 
is surjective, and so is

$$
\bigoplus_{i=1}^{N} H^{0}\left(X, \mathcal{F} \otimes P_{\xi} \otimes P_{\eta_{i}}\right) \otimes P_{\eta_{i}}^{\vee} \longrightarrow \mathcal{F} \otimes P_{\xi} .
$$

In other words, the map $(1)$ is surjective for $\left(\xi_{1}, \ldots, \xi_{N}\right)=\left(\xi+\eta_{1}, \ldots, \xi+\eta_{N}\right)$, for all $\xi \in \operatorname{Pic}^{0}(X)$. Choosing $\xi_{0}$ such that $h^{0}\left(X, \mathcal{F} \otimes P_{\xi_{0}+\eta_{i}}\right)$ takes the general (minimal) value for each $i$ in $\{1, \ldots, N\}$, we obtain that the map (1) is still surjective for $\left(\xi_{1}, \ldots, \xi_{N}\right)$ in a neighborhood of $\left(\xi_{0}+\eta_{1}, \ldots, \xi_{0}+\eta_{N}\right)$. This proves that $\mathcal{F}$ is continuously globally generated.

Corollary 3.2. - Let $X$ an irreducible projective variety with a finite map to an abelian variety. Any continuously globally generated coherent sheaf on $X$ is ample.

Proof. - Let $\mathcal{F}$ be a continuously globally generated coherent sheaf on $X$. By Proposition 3.1, there exists a connected abelian Galois étale cover $\pi: Y \rightarrow X$ such that $\pi^{*}\left(\mathcal{F} \otimes P_{\xi}\right)$ is globally generated for all $\xi \in \operatorname{Pic}^{0}(X)$.

Let $C$ be a curve in $Y$. If there is a trivial quotient $\pi^{*} \mathcal{F}_{\mid C} \rightarrow \mathcal{O}_{C}$, we have also surjections $\pi^{*}\left(\mathcal{F} \otimes P_{\xi}\right)_{\mid C} \rightarrow \pi^{*} P_{\xi \mid C}$ for each $\xi \in \operatorname{Pic}^{0}(X)$. Since $\pi^{*}\left(\mathcal{F} \otimes P_{\xi}\right)$ is globally generated, so is $\pi^{*} P_{\xi \mid C}$. This implies that the composition $\mathrm{Pic}^{0}(X) \rightarrow \mathrm{Pic}^{0}(Y) \rightarrow \mathrm{Pic}^{0}(C)$ is zero, hence that $\pi(C)$ is contracted by any map from $X$ to an abelian variety. This contradicts our hypothesis, hence $\pi^{*} \mathcal{F}_{\mid C}$ has no trivial quotient.

By Gieseker's Lemma, $\pi^{*} \mathcal{F}$ is ample, and so is $\mathcal{F}(\S 2)$.

\section{The main theorem}

Following [13, Definition 2.1], we say that a coherent sheaf $\mathcal{F}$ on an abelian variety $A$ is $M$-regular if

$$
\operatorname{codim}_{\mathrm{Pic}^{0}(A)} \operatorname{Supp}\left(R^{i} \widehat{\mathcal{S}}(\mathcal{F})\right)>i
$$

for all $i>0\left(R^{i} \widehat{\mathcal{S}}\right.$ is the $i$ th Fourier-Mukai functor). This is the case if

$$
\operatorname{codim}_{\mathrm{Pic}^{0}(A)}\left\{\xi \in \operatorname{Pic}^{0}(A) \mid H^{i}\left(A, \mathcal{F} \otimes P_{\xi}\right) \neq 0\right\}>i
$$

for all $i>0$. We refer to [12] and [13] for more details. For our purposes, the main result of [13] (Proposition 2.13) is that an $M$-regular coherent sheaf on an abelian variety is continuously globally generated.

THEOREM 4.1. - Let $X$ be a smooth connected projective variety with a finite map to a simple abelian variety, let $Y$ be a smooth connected projective variety with a finite surjective map $f: Y \rightarrow X$. If $f$ factors through no nontrivial connected abelian Galois étale covering of $X$, the vector bundle $E_{f} \otimes \omega_{X}$ is ample. 
Proof. - Let $n$ be the common dimension of $X$ and $Y$, and let $\alpha: X \rightarrow A$ be a finite map to a simple abelian variety such that $\operatorname{Pic}^{0}(\alpha): \operatorname{Pic}^{0}(A) \rightarrow \operatorname{Pic}^{0}(X)$ is injective. Set $g=\alpha \circ f$. By [4, Theorem 1], [5, Theorem 0.1], and [3, Remark 1.6] (see also [3, Theorem 1.2]), every irreducible component of the set

$$
V_{i}=\left\{\xi \in \operatorname{Pic}^{0}(A) \mid H^{n-i}\left(Y, g^{*} P_{\xi}^{\vee}\right) \neq 0\right\}
$$

is a translated abelian subvariety of $\operatorname{Pic}^{0}(A)$ of codimension at least $i$. In particular, since $A$ is simple, $V_{i}$ is finite for $i>0$.

Since $Y$ is connected, we have

$$
\begin{aligned}
V_{n} & =\left\{\xi \in \operatorname{Pic}^{0}(A) \mid H^{0}\left(Y, g^{*} P_{\xi}^{\vee}\right) \neq 0\right\} \\
& =\left\{\xi \in \operatorname{Pic}^{0}(A) \mid g^{*} P_{\xi}^{\vee} \simeq \mathcal{O}_{Y}\right\} \\
& =\operatorname{Ker}\left(\operatorname{Pic}^{0}(g): \operatorname{Pic}^{0}(A) \rightarrow \operatorname{Pic}^{0}(Y)\right),
\end{aligned}
$$

hence $V_{n}=\{0\}$ since both $\operatorname{Pic}^{0}(\alpha)$ and $\operatorname{Pic}^{0}(f)$ are injective $(f$ factors through no nontrivial abelian étale covering of $X)$. Consider now

$$
\begin{aligned}
W_{i} & =\left\{\xi \in \operatorname{Pic}^{0}(A) \mid H^{i}\left(X, E_{f} \otimes \omega_{X} \otimes \alpha^{*} P_{\xi}\right) \neq 0\right\} \\
& =\left\{\xi \in \operatorname{Pic}^{0}(A) \mid H^{i}\left(A, \alpha_{*}\left(E_{f} \otimes \omega_{X}\right) \otimes P_{\xi}\right) \neq 0\right\} .
\end{aligned}
$$

By Serre duality on $Y$,

$$
\begin{aligned}
V_{i} & =\left\{\xi \in \operatorname{Pic}^{0}(A) \mid H^{i}\left(Y, \omega_{Y} \otimes g^{*} P_{\xi}\right) \neq 0\right\} \\
& =\left\{\xi \in \operatorname{Pic}^{0}(A) \mid H^{i}\left(X, f_{*} \omega_{Y} \otimes \alpha^{*} P_{\xi}\right) \neq 0\right\} .
\end{aligned}
$$

Since $f_{*} \omega_{Y}=f_{*} \omega_{Y / X} \otimes \omega_{X}=\omega_{X} \oplus\left(E_{f} \otimes \omega_{X}\right)$, we have $W_{i} \subset V_{i}$ and $W_{n}=\varnothing$. It follows that $W_{i}$ is finite, hence $\operatorname{codim}\left(W_{i}\right)>i$ for each $i>0$, so that the sheaf $\alpha_{*}\left(E_{f} \otimes \omega_{X}\right)$ on $A$ is $M$-regular, hence continuously globally generated. It is therefore ample by Corollary 3.2, and, since $\alpha$ is finite, so are $\alpha^{*}\left(\alpha_{*}\left(E_{f} \otimes \omega_{X}\right)\right)$ and its quotient $E_{f} \otimes \omega_{X}(\S 2)$.

In the following remarks, we keep the hypotheses and notation of the theorem and its proof.

REMARK 4.2. - The proof of the theorem shows that the sheaf $\alpha_{*}\left(E_{f} \otimes \omega_{X}\right)$ is continuously globally generated. In particular, if $f$ is not an isomorphism, $E_{f} \otimes \omega_{X}$ has nonzero sections, hence $p_{g}(Y)>p_{g}(X)$.

REMARK 4.3. - The simplicity of the abelian variety in the theorem is essential: if $B$ is an abelian variety and $g=\left(f, \operatorname{Id}_{B}\right): Y \times B \rightarrow X \times B$, we have $E_{g}=p^{*} E_{f}$, where $p: X \times B \rightarrow X$ is the first projection, hence $E_{g} \otimes \omega_{X \times B}=p^{*}\left(E_{f} \otimes \omega_{X}\right)$ is not ample if $B$ is nonzero. The locus $W_{i}$ for $g$ contains $\operatorname{Pic}^{0}(A) \times\{0\}$ for $i \leq \operatorname{dim}(B)$; in particular, for $i=\operatorname{dim}(B)$, it is an abelian subvariety of codimension $i$ of $\operatorname{Pic}^{0}(A \times B)$.

Note however that if $A$ is not simple but $\alpha(X)$ is not ruled by nonzero abelian subvarieties of $A$, the end of the proof of Theorem 3 of [3] implies $\operatorname{codim}\left(V_{i}\right)>i$

TOME $134-2006-\mathrm{N}^{\mathrm{O}} 2$ 
for each $i>0$. The proof above shows that the conclusion of Theorem 4.1 still holds.

REMARK 4.4. - If $X$ is not an abelian variety, $\omega_{X}$ is already ample (see, e.g., [1, Théorème 6.9]) and one can show that the hypothesis that $f$ does not factor through a nontrivial connected abelian Galois étale covering of $X$ is unnecessary. If $X$ is a (simple) abelian variety, any finite cover $Y \rightarrow X$ factorizes as $Y \stackrel{f}{\rightarrow} X^{\prime} \stackrel{\rho}{\rightarrow} X$ where $\rho$ is an isogeny and $f$ satisfies the hypotheses of the theorem.

Remark 4.5. - Assume $X=A$ and let $d$ be the degree of $f$. We want to prove that for all $i \geq d-1$, the set $W_{i}$ is empty, i.e.,

$$
H^{i}\left(A, E_{f} \otimes P_{\xi}\right)=0 \quad \text { for all } \xi \in \operatorname{Pic}^{0}(A) .
$$

By a theorem of Simpson [15], all points of $V_{i}$, hence a fortiori all points of $W_{i}$, are torsion points. As explained in the introduction, Theorem 4.1 implies that the morphism

$$
H^{n-i}(f, \mathbb{C}): H^{n-i}(A, \mathbb{C}) \longrightarrow H^{n-i}(Y, \mathbb{C})
$$

is bijective for $i \geq d-1$. Using the Hodge decomposition, this implies $0 \notin W_{i}$. For any isogeny $\pi: A^{\prime} \rightarrow A$, the smooth variety $Y^{\prime}=Y \times_{A} A^{\prime}$ is connected and if $f^{\prime}: Y^{\prime} \rightarrow A^{\prime}$ is the second projection, we have $E_{f^{\prime}}=\pi^{*} E_{f}$. It follows that for $i \geq \operatorname{deg}\left(f^{\prime}\right)-1=d-1$, we have

$$
0=H^{i}\left(A^{\prime}, E_{f^{\prime}}\right)=H^{i}\left(A, E_{f} \otimes \pi_{*} \mathcal{O}_{A^{\prime}}\right)=\bigoplus_{\xi \in \operatorname{Ker}\left(\operatorname{Pic}^{0}(\pi)\right)} H^{i}\left(A, E_{f} \otimes P_{\xi}\right) .
$$

In particular, $W_{i}$ contains no torsion points, hence is empty.

Equality (2) does not hold in general for $0 \leq i<d-1$, as shown by the following example. Take an elliptic curve $C$, with origin $o_{C}$. Let $L$ be a very ample line bundle on $A$ and let $Y \subset C \times A$ be a general (smooth) element of $\left|\mathcal{O}_{C}\left((n+1) o_{C}\right) \otimes L\right|$. Following the proof of [10, Lemma 6.3.43], one sees that the second projection $f: Y \rightarrow A$ is finite (of degree $d=n+1$ ). By the Lefschetz theorem, the induced morphism

$$
H^{n-i}\left(C \times A, \mathcal{O}_{C \times A}\right) \longrightarrow H^{n-i}\left(Y, \mathcal{O}_{Y}\right)
$$

is bijective for $i>0$ and injective for $i=0$. In particular, $H^{n-i}(f, \mathcal{O})$ is not surjective for $0 \leq i<n$, hence $0 \in W_{i}$, i.e.,

$$
H^{i}\left(A, E_{f}\right) \neq 0 \text { for } 0 \leq i<d-1=n .
$$

In particular, $H^{n-1}\left(A, E_{f}\right) \neq 0$, and it follows from [12, Proposition 2.7], that the $M$-regular vector bundle $E_{f}$ does not satisfy Mukai's condition $\mathrm{WIT}_{0}$ when $n>1$ (sheaves that satisfy condition $\mathrm{WIT}_{0}$ are $M$-regular). 


\section{BIBLIOGRAPHY}

[1] Debarre (O.) - Théorèmes de connexité et variétés abéliennes, Amer. J. Math., t. 117 (1995), pp. 787-805.

[2] — Tores et variétés abéliennes complexes, in Cours spécialisés, vol. 6 , Société Math. France, Paris ; EDP Sciences, Les Ulis, 1999.

[3] Ein (L.) \& Lazarsfeld (R.) - Singularities of theta divisors and the birational geometry of irregular varieties, J. Amer. Math. Soc., t. 10 (1997), pp. 243-258.

[4] Green (M.) \& Lazarsfeld (R.) - Deformation theory, generic vanishing theorems, and some conjectures of Enriques, Catanese and Beauville, Invent. Math., t. 90 (1987), pp. 389-407.

[5] Higher obstructions to deforming cohomology groups of line bundles, J. Amer. Math. Soc., t. 4 (1991), pp. 87-103.

[6] Hwang (J.-M.), Kebekus (S.) \& Peternell (T.) - Holomorphic maps onto varieties of non-negative Kodaira dimension, J. Algebraic Geom., t. 15 (2006), pp. 551-561.

[7] Kim (M.) \& MAniel (L.) - On branched coverings of some homogeneous spaces, Topology, t. 38 (1999), pp. 1141-1160.

[8] Kubota (K.) - Ample sheaves, J. Fac. Sci. Univ. Tokyo Sect. I A Math., t. 17 (1970), pp. 421-430.

[9] LAzARSFeld (R.) - A Barth-type theorem for branched coverings of projective space, Math. Ann., t. 249 (1980), pp. 153-162.

[10] _ Positivity in algebraic geometry II, in Ergebnisse der Mathematik und ihrer Grenzgebiete, vol. 49, Springer-Verlag, Heidelberg, 2004.

[11] Manivel (L.) - Vanishing theorems for branched coverings of projective space, Invent. Math., t. 127 (1997), pp. 401-416.

[12] Mukai (S.) - Duality between $D(X)$ and $D(\widehat{X})$ with its application to Picard sheaves, Nagoya Math. J., t. 81 (1981), pp. 153-175.

[13] Pareschi (G.) \& Popa (M.) - Regularity on abelian varieties I, J. Amer. Math. Soc., t. 16 (2003), pp. 285-302.

[14] Peternell (T.) \& Sommese (A.) - Ample Vector Bundles and Branched Coverings, II, in The Fano Conference (Collino(A.), Conte (A.) \& Marchiso (M.), eds.), Univ. Torino, 2004, pp. 625-645.

[15] Simpson (C.) - Subspaces of moduli spaces of rank one local systems, Ann. Sci. École Norm. Sup., t. 26 (1993), pp. 361-401. 